\title{
Ein Beitrag zur spätglazialen Vegetations- und Waldentwicklung im westlichen Salzachgletschergebiet
}

\author{
Von Hans Schmeide, Bernau/Chiemsee
}

Mit 5 Abbildungen

$\mathrm{Zusammenfassung.} \mathrm{Von} \mathrm{zwei} \mathrm{spätglazialen} \mathrm{Seen} \mathrm{in} \mathrm{der} \mathrm{westlichen} \mathrm{Drumlinland-}$ schaft $(440 \mathrm{~m})$ und der westlichen Randmoränenlandschaft $(600 \mathrm{~m})$ des Salzachgletschers und aus einem Moor aus dem ehemals unvereisten Gebiet $(700 \mathrm{~m})$ zwischen Salzach- und Chiemseegletscher wurden die Sedimente und Torfe pollenanalytisch untersucht.

Die Vegetationsentwicklung beginnt mit Pioniergesellschaften. Die Wiederbewaldung wird durch eine Salix-Juniperus-Hippophaë-Phase eingeleitet, der ein Baumbirkenvorstoß folgt. Der eindeutige Nachweis der älteren Tundrenzeit (I c) war nicht möglich. Im Alleröd waren geschlossene Wälder vorhanden. Die jüngere Tundrenzeit (III) war in $700 \mathrm{~m}$ Höhe einwandfrei nachzuweisen. Es kam zu einer starken Lichtung der Wälder. In den beiden Seeprofilen trat gegen Ende des Alleröds eine Störung in den Ablagerungen auf.

Eine C14-Datierung zeigt eine Differenz von 600-700 Jahren, deren Ursache nicht geklärt werden konnte.

Die Vegetations- und Waldentwicklung zeigt mit anderen Gebieten eine gute Ubereinstimmung.

Summary. Sediments and paet were pollenanalytical investigated: 1 . from one late glacial lakes in the western Drumlin area, 2. one late glacial lakes in the brim of the western moraine of the Salzach glacier, 3. from a paet moos situated between the Salzach- and Chiemsee-glacier.

The evolution of the vegetation starts with pionier associations. The reforestation is indroduced by the Salix-Juniperus-Hippophaë-phase and succeeded by birch-trees. An indentivication of the older Dryas-time (I c) is not possible. In the Alleröd-time existed dense forests. The younger Dryas (III) can be definithy proved in an altidude of 700 meters the forests receded. An interruption in the depositions of the both profiles of the lakes can be seen at the end of the Alleröd.

A radiocarbon determination shows a difference from 600-700 years, which can not be explained.

The evolution of vegetation and forest shows a good conformity with other regions.

\section{Einleitung}

Der Ablauf der spätglazialen Vegetationsentwicklung im nördlichen Mitteleuropa, im südwestdeutschen Alpenvorland und in den Südalpen ist in den letzten Jahren durch pollenanalytische Untersuchungen weitgehend bekannt geworden. Auch die Altersstellung der einzelnen Vegetationsabschnitte ist für diese Gebiete gesichert. Durch die Arbeiten von Firbas (1923), Paul und Ruoff (1927) und Mayer (1964, 1966) sind wir zwar über die postglaziale Waldentwicklung im südostdeutschen Alpenvorland im großen Rahmen unterrichtet, über die spätglaziale Vegetations- und Klimaentwicklung geben diese Arbeiten aber nur wenig Auskunft.

Für das gesamte nordöstliche Alpenvorland liegen für das Spätglazial nur vereinzelte Untersuchungen vor. Es sind dies die Arbeit von Firbas (1934) über das Spätglazial im Bereich des ehemaligen Rosenheimer Sees, die Untersuchungen von MAYER (1966) im Böckelweiher bei Berchtesgaden, die im Egelseegebiet, nordöstlich von Salzburg, von Lürzer (1954), die im Ibmer Moos (Ober-Österreich) von Prammer-Gräflinger (in Gams 1947), die aus dem Filzmoos von Krisai (1961) und vom Lunzer See von Gams (1927) und Burger (1964). Diese wenigen Arbeiten zwischen Iller und östlichem Alpenrand lassen ein dichteres Netz von Untersuchungen wünschenswert erscheinen. Für das 
Gebiet des ehemaligen Rosenheimer Sees liegt eine Neubearbeitung von BEUG vor. Ein weiterer Beitrag soll die vorliegende Arbeit sein, deren Untersuchungen sich auf das westl. Salzachvorlandgletschergebiet beschränken und zwar auf Ablagerungen innerhalb und außerhalb der westl. Randmoräne und auf das westl. Drumlinfeld. ${ }^{1}$ )

\section{Zur Methodik}

Die beiden Profile aus den spätglazialen Seen wurden als überlappende Bohrkerne mit der verbesserten Dachnowsky-Sonde erbohrt. Das Profil aus dem Moor der Pechschnait wurde dagegen in Form von Prismen bis in den mineralischen Untergrund an einer Stichwand herausgeschnitten.

Die Aufbereitung der einzelnen Proben erfolgte nach dem durch Troels-Sмiтн (1954) verbesserten Acetolyseverfahren nach ErdtManN. Die tonhaltigen Proben wurden mit kalter HF behandelt. Obwohl für die ältesten Ablagerungen teilweise ein enger Probenabstand gewählt wurde, zeigte es sich im Laufe der Untersuchung, daß bei Torfablagerungen auch oft ein Probenabstand von unter $1 \mathrm{~cm}$ gewählt werden müßte, um die spätglaziale Entwicklung einwandfrei $\mathrm{zu}$ erfassen.

Die Pollen-, Torf- und limnischen Sedimentsignaturen (Abb. 2), sowie die Zoneneinteilung erfolgten nach FiRBaS (1949). Zum Vergleich der einzelnen Diagramme wurden dieselben in lokale Abschnitte $(\mathrm{a}-\mathrm{m})$ unterteilt.

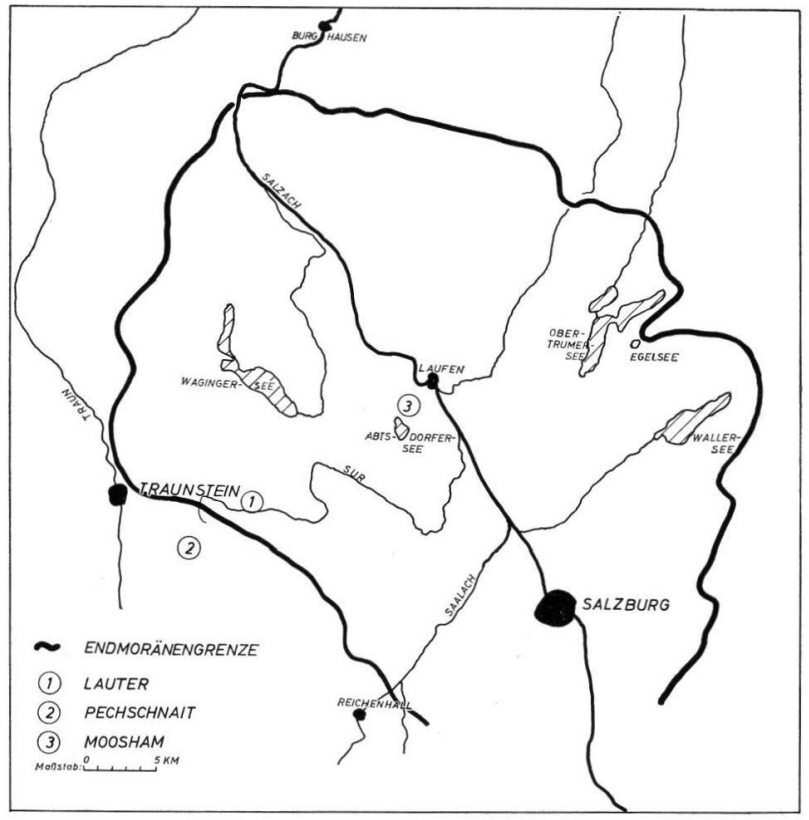

Abb. 1. Das Untersuchungsgebiet mit den spätglazialen Ablagerungen (1 Lauter, 2 Pechschnait, 3 Moosham).

\section{Das Untersuchungsgebiet}

Die Untersuchungen erstrecken sich im westlichen Salzachvorlandgletschergebiet auf die Ablagerungen eines kleinen Endmoränensees bei Lauter (östl. von Traunstein) in $600 \mathrm{~m}$ NN und eines ehemals kleinen Sees in der Drumlinlandschaft bei Moosham östlich

1) Herrn Dr. Ziegler vom geologischen Landesamt München möchte ich für die Diskussion während der gemeinsamen Geländebegehung danken und Herrn Dr. Ganss von demselben Amt für die Vermittlung der C14-Datierung, die in dankenswerter Weise Herr Dr. GEY vom Niedersächsischen Landesamt für Bodenforschung, Hannover, durchgeführt hat. 
von Laufen in $440 \mathrm{~m}$ ü. NN. Ein weiteres Profil entstammt dem Moor in der Pechschnait. Dieses liegt nur wenige $\mathrm{km}$ südwestlich von Lauter im ehemals unvereisten $\mathrm{Ge}-$ biet zwischen Salzach und Chiemseegletscher in einer Höhe von 690 m NN (Abb. 1).

Klimadaten für das Untersuchungsgebiet bei Lauter und der Pechschnait liegen vor von Traunstein $(580 \mathrm{~m})$, Oberteisendorf $(522 \mathrm{~m})$ und Achtal $(580 \mathrm{~m})$. Das Gebiet liegt noch im Stau der
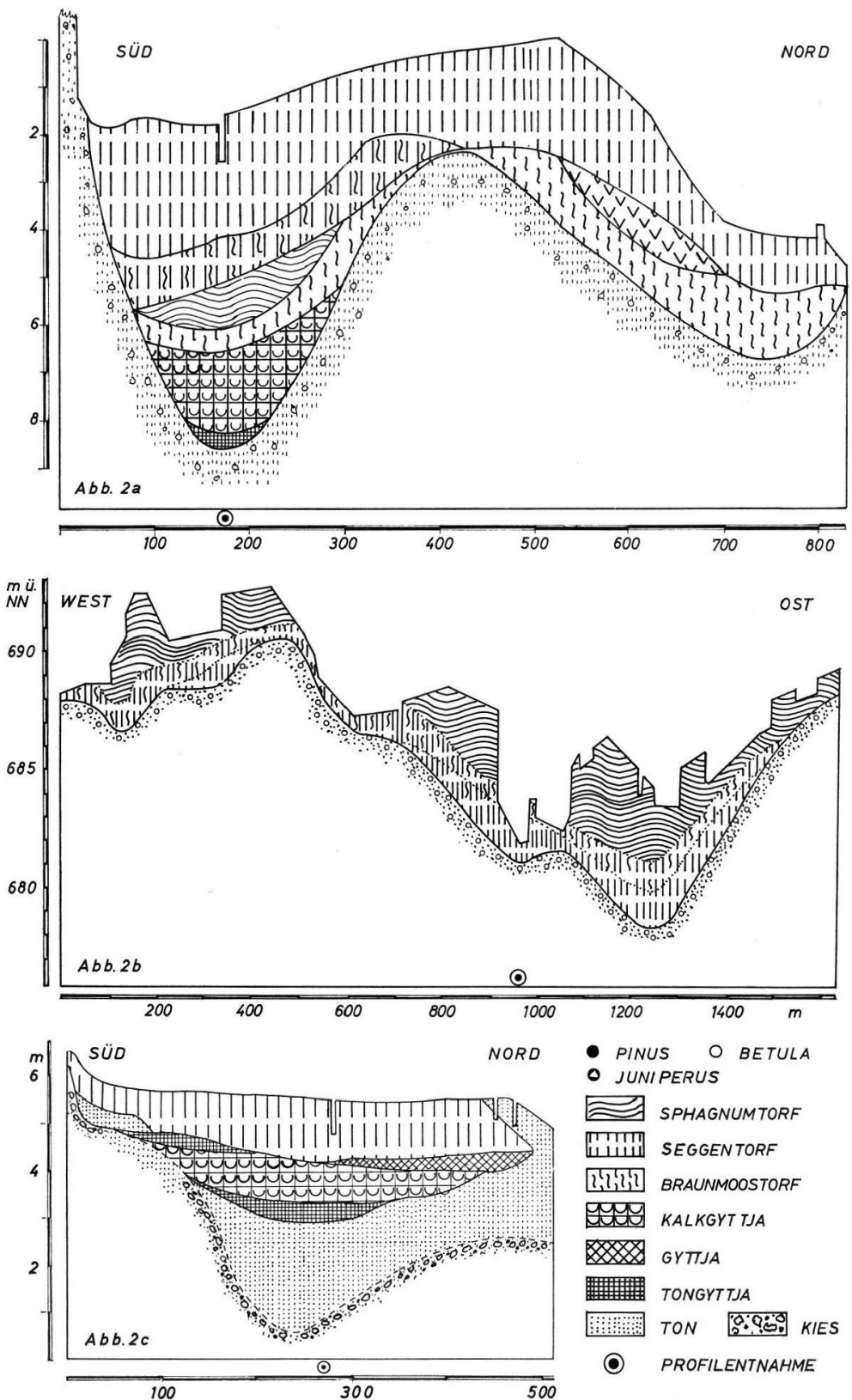

- pinus o betula

- juniperUs

ॠ SPHAGNUMTORF

Wiiil SEGGENTORF

L,T,?,? BRAUNMOOSTORF

gyYg KALKGYTTJA

GYTTA

TONGYTTJA

TON TO:PEO KIES

- PROFILENTNAHME

Abb. 2. Stratigraphische Profile durch die Moor- und Seeablagerungen in Lauter (a), in der Pechschnait (b) und bei Moosham (c). 
nördlichen Voralpen, und die Niederschläge sind entsprechend hoch. Traunstein verzeichnet im langjährigen Mittel (1891-1960) $1532 \mathrm{~mm}$, Oberteisendorf (1891-1930) $1507 \mathrm{~mm}$ und Achtal (1891-1960) sogar $1595 \mathrm{~mm}$. Die mittlere Jahrestemperatur liegt in Traunstein bei $7,4^{\circ} \mathrm{C}$, das Januarmittel bei $-2^{\circ} \mathrm{C}$ und das Julimittel bei $16,9^{\circ} \mathrm{C}$. Für das Gebiet der Drumlinlandschaft stehen die Niederschlagswerte von Laufen und Kirchanschöring zur Verfügung. Laufen verzeichnet im langjährigen Mittel (1891-1960) $1158 \mathrm{~mm}$ und Kirchanschöring (1891-1930) $965 \mathrm{~mm}$ pro Jahr. Die Temperaturwerte können nur auf Grund der vorhandenen Werte von Burghausen und Trostberg interpoliert werden. Das Jahresmittel liegt für das Gebiet um den Abtsdorfer See bei $7,6^{\circ}$ C. (Ber. d. D. W. D. Nr. 17 und Mitteil. d. D. W. D. Nr. 4.)

\section{Ergebnisse der Untersuchungen}

\section{a) L a g e}

\section{Lauterer Filzen}

Nördlich der Bahnlinie Traunstein-Salzburg, ca. $5 \mathrm{~km}$ von Traunstein entfernt, liegt nordöstlich der Ortschaft Lauter, innerhalb der ersten Rückzugstadien der äußeren Randmoräne, der Lauterer Filz. Große Teile des im zentralen Teil als Hochmoor ausgebildeten Moores sind heute schon abgetorft oder bewaldet. Niedermoorartige Randpartien werden teilweise landwirtschaftlich genutzt. Durch ein von Südwest nach Nordost verlaufendes Bohrprofil (Abb. 2a), das die Abt. Moorkunde und Torfwirtschaft der Bayer. Landesanstalt für Bodenkultur, Pflanzenbau und Pflanzenschutz (siehe Vidal u. Hohenstatter) durch die Lauterer Filzen gelegt hatte, wurde die Wahl des Bohrpunktes für das untersuchte Spätglazialprofil bestimmt. Zwischen steilen Moränenhügeln zieht sich von der Ortschaft Lauter in nordöstlicher Richtung ein heute als Grünland genutztes Niedermoor. Unter $560 \mathrm{~cm}$ Niedermoor- und Bruchtorf liegen limnische Ablagerungen eines kleinen spätglazialen Endmoränensees, der gegen Ende des Alleröd durch Hypnum-Schwingrasen verlandete. Die den limnischen Ablagerungen aufliegenden Torfschichten zeigen Störungen. Das von der Bayer. Landesanstalt erbohrte Profil läßt eine Aufwölbung der Seekreide und der darüberliegenden Hypnumtorfschichten erkennen, die wahrscheinlich durch die aufliegenden Niedermoorschichten verursacht wurde. Das Profil wurde ca. $500 \mathrm{~m}$ nordöstlich von Lauter, ungefähr in der Mitte des ehemaligen Sees erbohrt.

b) Stratigraphie

Das Profil umfaßt nur die limnischen Ablagerungen, die darüberliegenden Torfschichten wurden nicht untersucht.

$530-560 \mathrm{~cm}$ dkl.braune Grobdetritusgyttja, kiefern-birkenzeitlich, die bei $550 \mathrm{~cm}$ eine wärmezeitliche Schicht mit hohen EMW-Werten eingelagert hat. An Makrofossilien waren vorhanden: unbestimmbare Braunmoosreste, Scorpidium-Blättchen, Sphagnum-Blättchen der Cymbifoliagruppe und bei $530-540 \mathrm{~cm}$ Holzkohle und Flugasche.

$560-600 \mathrm{~cm}$ grauweiße Kalkgyttja mit Conchylienresten, dünne Lagen von Braunmoosresten, Scorpidium-Blättchen. Oogonien von Chara und bei 570 Holzkohle, bei 580 Flugasche.

$600-620 \mathrm{~cm}$ grauweiße Kalkgyttja mit Chonchylien, Braunmoosfragmenten, Carex-Radizellen, Gramineen-Epidermis, Chara-Oogonien.

$620-640 \mathrm{~cm}$ grauweiße Kalkgyttja mit Braunmoos- und Scorpidium-Blattresten.

$640-675 \mathrm{~cm}$ gelbgraue Kalkgyttja mit wenig Chonchylien, ab $645 \mathrm{~cm}$ mit toniger Beimengung (HF), bei $660 \mathrm{~cm}$ Ubergang in eine rötliche und anschließend grünliche tonige Kalkgyttja. Einzelne Braunmoosblattreste.

675-692 cm stark kalkhaltige, grüne Tongyttja mit einzelnen Schnecken, Sphagnumblattresten, Radizellen, Braunmoosfragmente sowie unbestimmbare Blattstückchen.

$692-709 \mathrm{~cm}$ schwach kalkhaltige, dkl.grüne Tongyttja mit wenig organischer Substanz. CharaOogonien und Pediastren.

$709-720 \mathrm{~cm}$ dkl.grüne Tongyttja, bei $720 \mathrm{~cm}$ schwach kalkhaltig. Einlagerung von $\mathrm{FeS}_{2}$ (Pyrit). $720-740 \mathrm{~cm}$ dkl.grüne, kalkfreie Tongyttja, einzelne Sphagnum-Blattreste und Radizellen.

8 Eiszeitalter u. Gegenwart 
$740-760 \mathrm{~cm}$ sandiger, grauer Ton mit Feinkies, Pyritkügelchen, Pediastren und Chitinreste, Sekundärpollen.

Makroreste, die im Diagramm eingetragen sind, wurden hier fortgelassen.

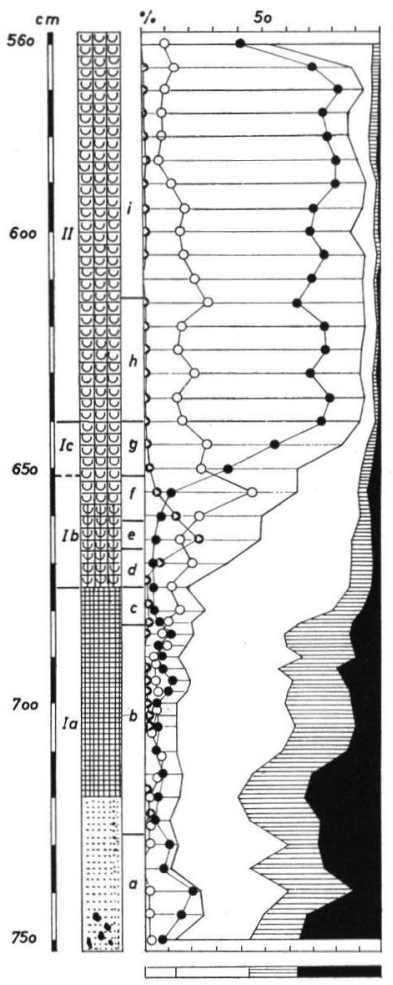

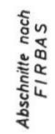

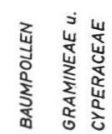

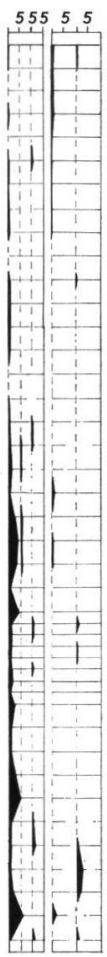

$\stackrel{10,30, \quad 10,30}{2}$
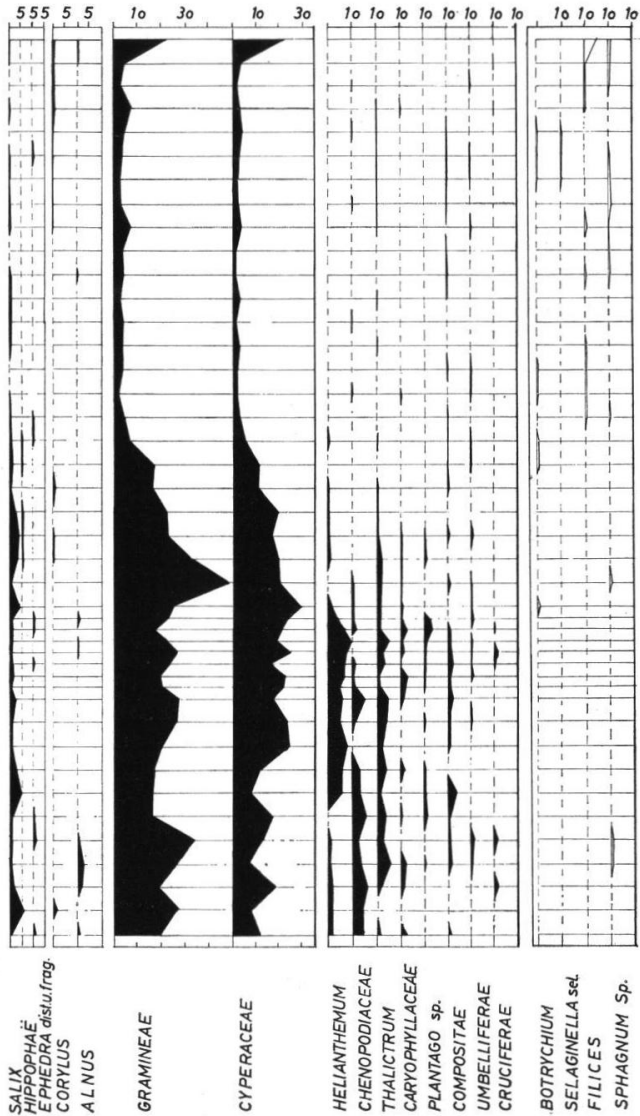
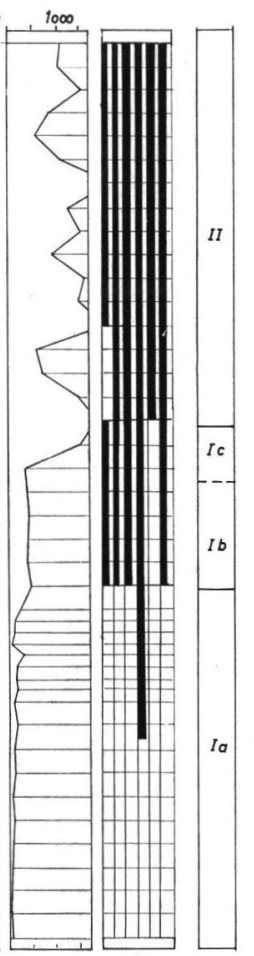

Abb. 3. Pollen- und Sporendiagramm des Spätglazials von Lauter.

Als Gesamtdiagramm berechnet.

c) Vegetationsentwicklung

Um eine Koordinierung einzelner Vegetationsabschnitte mit anderen Profilen zu ermöglichen, wurden, wie schon erwähnt, die Diagramme zunächst in lokale Abschnitte gegliedert. Vom eisfreiwerden bis zur Zeit der geschlossenen Bewaldung ergibt sich folgendes Bild der Vegetationsentwicklung im Gebiet von Lauter (Abb. 3):

Waldlose Z eit. Mit sehr hohen NBP-Werten von $85-90 \%$ setzt das Diagramm ein. Die geringe Pollendichte (PD), die Zusammensetzung des NBP-Anteils und das Sediment lassen schließen, daß baumlose, artenreiche Gesellschaften vorherrschten. Die Pinus-Pollenwerte sind auf Fernflug zurückzuführen, während Zwergbirken und Zwergweiden wahrscheinlich schon im Gebiet vertreten waren. Stratigraphisch ist die Zeit gekennzeichnet durch die Sedimentation eines grauen, sandigen Tones und einer grau-grünen Tongyttja, in der Pyritkügelchen eingelagert sind. Eine Untergliederung in die Abschnitte $a-c$ wurde für diese Zeit vorgenommen.

A bschnitt a: Nach dem Eisrückgang wurden zunächst sandige Tone, die vereinzelt noch Feinkies aufweisen, abgelagert. Der Pollenniederschlag ist gering, und die in diesen $\mathrm{Ab}-$ schnitten stärker vertretenen Pinus-Pollen dürften z. T. umgelagertem, älterem Material entstammen, da neben stark korrodierten Alnus-Pollen, die einwandfrei als Sekundärpollen anzu- 
sehen sind, auch ein Pollenkorn von Pterocarya gefunden wurde. Zum anderen Teil handelt es sich wahrscheinlich um Ferntransporte aus dem südlichen Alpenvorland durch Föhn. Unter den NBP dominieren Gramineen und Artemisia (bis 35\%); Chenopodiaceen und Thalictrum erreichen verhältnismäßig hohe Werte, während Helianthemum $\mathrm{cf}$. alpestre erst in der Ausdehnung begriffen ist. Ephedra ist in diesem Abschnitt auch schon vertreten.

A b s chnitt b: Im Baumpollenbild treten Salix und Betula oder Juniperus stärker hervor. Die NBP haben immer noch einen hohen Anteil. Gramineen und Artemisia sind stark verbreitet. Neben Chenopodiaceen, Thalictrum und Charyophyllaceen tritt jetzt Helianthemum stärker hervor und erreicht im weiteren Verlauf seine Dominanz. Die Cyperaceenwerte steigen auch an, und mit dem Rückgang von Artemisia gegen Ende des Abschnittes ist eine stärkere Ausbreitung von Gramineen, Cyperaceen, sowie der Strauchbirken verbunden. Betula nana scheint sich ausgebreitet zu haben, denn eine Zunahme der Pollendichte, sowie Funde von Großresten in Form von Blattfragmenten, Holzteilchen und Knospenschuppen, zeigen das Vorhandensein der Birke in der Nähe des Sees an. Der leichte Anstieg der Pinuskurve könnte vielleicht als eine vorübergehende Ausbreitung von Pinus montana gedeutet werden, da die NBP-Werte zurückgehen.

A bschnitt c: Er ist gekennzeichnet durch den Vorstoß von Strauchbirken und einem Rückgang von Pinus bei noch hohen NBP-Werten. Während Artemisia zurückgeht, findet die Beteiligung von Helianthemum an den Pioniergesellschaften in diesem Abschnitt ein Ende. Dafür breiten sich aber die Gräser aus; ebenso erscheinen wieder Ephedra und Selaginella sel..

Juniperus-Zeit, Abschnitt d: Mit Beginn dieses Abschnittes kommt es zu einer Sedimentationsänderung. An Stelle der Tongyttja tritt tonhaltige Kalkgyttja. Die beginnende Bewaldung wird durch einen starken Rückgang der NBP-Werte und eine Ausbreitung von Juniperus angezeigt. Holzreste und Fruchtschuppen von Betula pubescens zeigen schon das Vorhandensein der Baumbirken. Hippophaë ist auch vertreten, hat aber im Gebiet keine nennenswerten Bestände gebildet. Die Strauch- und Baumbestände waren noch nicht geschlossen, da die Gramineen- und Cyperaceenwerte noch sehr hoch und Vertreter der Pioniergesellschaften auch noch vorhanden sind.

A bschnitt e : Er umfaßt die Zeit des Juniperusgipfels bei gleichzeitigem Rückgang der Birke und einem weiteren Zurückgehen der NBP-Werte.

B e tula-Zeit, Abschnitt f: Rinden- und Fruchtreste zeigen an, daß sich Baumbirken und Kiefern weiter ausbreiten, wobei auch die vorhandenen Wacholder- und Sanddornbestände verdrängt werden. Betula nana scheint aber noch vertreten gewesen zu sein, da deren Großreste in den Ablagerungen dieses Abschnittes vorhanden sind. Die Gramineen- und Cyperaceenbestände werden durch die vorrückende Bewaldung allmählich verdrängt. Das Ende des $\mathrm{Ab}$ schnittes ist gekennzeichnet durch den Rückgang der NBP von 40 auf $20 \%$ und außerdem durch den Ubergang der tonigen Kalkgyttja in eine fast tonfreie.

B e tula-Pinus-Zeit, Abschnit g: Ein rascher Anstieg der Pinuskurve und die Pinus-Dominanz kennzeichnen diesen Abschnitt. Betula und besonders die vorhandenen Juniperusbestände werden zurückgedrängt. Da Großreste von Pinus und Betula häufig sind, müssen beide Bäume im Untersuchungsgebiet bestandsbildend gewesen sein. Die NBP-Werte sind zu dieser Zeit bis unter $10 \%$ abgesunken.

Pinus-Betula-Zeit, Abschnitt h: In ihm kommt es zu einem kurzzeitigen Vorstoß von Baumbirken und einem sporadischen Auftreten von Juniperus. Die NBP-Werte sind gering $(10-15 \%)$, und die Pollendichte ist hoch.

A bschnitt i: Zunächst dominiert noch Pinus, Juniperus ist aber stärker vertreten. Die Birkenkurve geht zurück und bei den NBP ist zweimal ein leichter Anstieg zu verzeichnen, der mit dem Auftreten von Ephedra, Botrychium und Selaginella sel. zusammenfällt, so daß eine Auflockerung der geschlossenen Bestände eingetreten sein muß. Gegen Ende des Abschnittes, der mit einem Facieswechsel Kalkgyttja-Torf zusammenfällt, steigen die Gramineen- und Cyperaceenwerte an. Zu gleicher Zeit erscheinen aber in den Spektren Pollen von Corylus und Alnus.

\section{Pechschnait}

a) L a g e

Auf einem tertiären Höhenrücken nördlich des Teisenberges liegt ungefähr $3 \mathrm{~km}$ südöstlich von Traunstein entfernt, im ehemals eisfreien Gebiet zwischen Salzach- und Chiemseegletscher, das Moor in der Pechschnait. Es ist mit ca. 60 ha das größte unter einer Anzahl von Mooren, die auf dem Rücken außerhalb der von Nordwest nach Südost verlaufenden Endmoräne liegen. Die heutige Oberfläche des Moores liegt bei $690 \mathrm{~m}$ 
NN und damit 90 Meter höher als die nur einige Kilometer entfernten Lauterer-Filzen. Bei dem heute schon stark zerstörten Hochmoor handelt es sich um ein Hangmoor (Abb. 2b), das wahrscheinlich aus mehreren Zentren entstanden ist, die im Laufe der Zeit $\mathrm{zu}$ einem einzigen Moorkomplex zusammengewachsen sind. Soweit eine ursprüngliche Oberfläche noch vorhanden, ist sie verheidet oder mit Kiefern und Fichten bestockt. Nur im östlichen Teil ist noch ein kleiner Rest der ursprünglichen Vegetation in Form eines Latschenbestandes vorhanden, der aber auch schon eine regressive Entwicklung erkennen läßt. Die Moormächtigkeit beträgt im stark gesackten, zentralen Teil 6-7 Meter, wobei die Hochmoorschichten bis 4 Meter mächtig sein können (Vidal und Hohenstatter). Die Pechschnait ist eines der wenigen Moore des Alpenvorlandes in dem Dopplerit vorkommt. Die Doppleritadern können eine Stärke von über $10 \mathrm{~cm}$ erreichen und sind in einigen Torfstichen anzutreffen. Entwässert wird das Moor nach Nordosten durch den tief eingeschnittenen Stein- oder Sparzer-Graben und durch den Ammersbach nach Süden.

\section{b) Stratigraphie}

Das untersuchte Profil entstammt dem zentralen Teil des Moores und umfaßt die Spät- und älteste Postglazialzeit und ist ein Teil eines aus der gesamten Tiefe des Moores gewonnenen Profils. Die Tiefenangaben beziehen sich auf die heute stark gesackte Oberfläche des Moores.

$340-425 \mathrm{~cm}$ dunkelbrauner Seggentorf.

$425-450 \mathrm{~cm}$ dunkelbrauner, stark zersetzter Braunmoostorf; bei $445 \mathrm{~cm}$ Baumbirkenhorizont. $450-475 \mathrm{~cm}$ stark toniger Braunmoostorf.

$475-500 \mathrm{~cm}$ Tonmudde mit Braunmoosresten. Untersucht wurden nur die obersten $6 \mathrm{~cm}$.

ab $500 \mathrm{~cm}$ blaugrauer Ton, fossilfrei.

\section{c) Vegetationsentwicklung}

Das Profil läßt sich in folgende Abschnitte einteilen (Abb. 4):

Waldlose Zeit, Abschnitt c: Das Profil setzt erst im jüngsten Abschnitt der waldlosen Zeit ein und ist gekennzeichnet durch hohe NBP-Werte, die zwischen 80 und $50 \%$ liegen. Weiden sind stärker vertreten, und auch Ephedra ist vorhanden. Gramineen- und Cyperaceen-reiche Pioniergesellschaften mit Artemisia, Caryophyllaceen und Thalictrum herrschen vor. Da die Pinuswerte bei über 20\% liegen, sind vermutlich Kiefern in der Nähe des Moores schon vorhanden gewesen. Die Dominanz der Birke ist wahrscheinlich auf Strauchbirken zurückzuführen.

P inus-Juniperus-Zeit. Sie umfaßt die Abschnitte d, e, f wie in Lauter.

A bschnitt d: Die NBP-Werte sind bis auf $50 \%$ zurückgegangen, Artemisia tritt zurück, und Juniperus breitet sich aus.

A bschnitt e: Juniperus dominiert mit $13 \%$, Artemisia und die anderen Pionierpflanzen sind vollständig zurückgedrängt. Wie die beiden vorangegangenen Abschnitte ist auch dieser in der Pechschnait sehr zusammengedrängt. Ein kleinerer Probenabstand (von $1 \mathrm{~cm}$ ) hätte wahrscheinlich ein klareres Bild ergeben.

A bschnitt $f$ : Die in Lauter deutlich ausgeprägte Ausbreitung der Baumbirken anschließend an die Juniperus-Dominanz ist hier nur angedeutet. Spärlich vertreten ist auch Hippophaë. Während die Gräser- und Kräuterbestände zurückgehen, breitet sich die Kiefer kräftig aus.

$P$ inus-Zeit, Abschnitt g: Die NBP-Werte betragen $50-25 \%$, so daß wir mit einer geschlossenen Bewaldung, in der die Kiefer dominiert, erst gegen Ende des Abschnittes rechnen dürfen. Die Birke tritt im Gegensatz zu Lauter hier fast nicht hervor.

Abschnitt h: Neben der Kiefer breitet sich nun die Birke aus, wobei die ansteigenden Gramineen- und Kräuter-Werte auf eine leichte Lichtung der vorhandenen Waldbestände hinweisen.

Abschnitt $i$ : Die Birke hat zumindest an einigen Stellen das Moor besiedelt (Holzhorizont zur Zeit des Birkengipfels). Ebenso wie in Lauter erscheinen in diesem Abschnitt Pollen von Corylus und Alnus. Da die Pollenwerte sehr gering sind, muß ihr Auftreten durch Fernflug bedingt sein. 


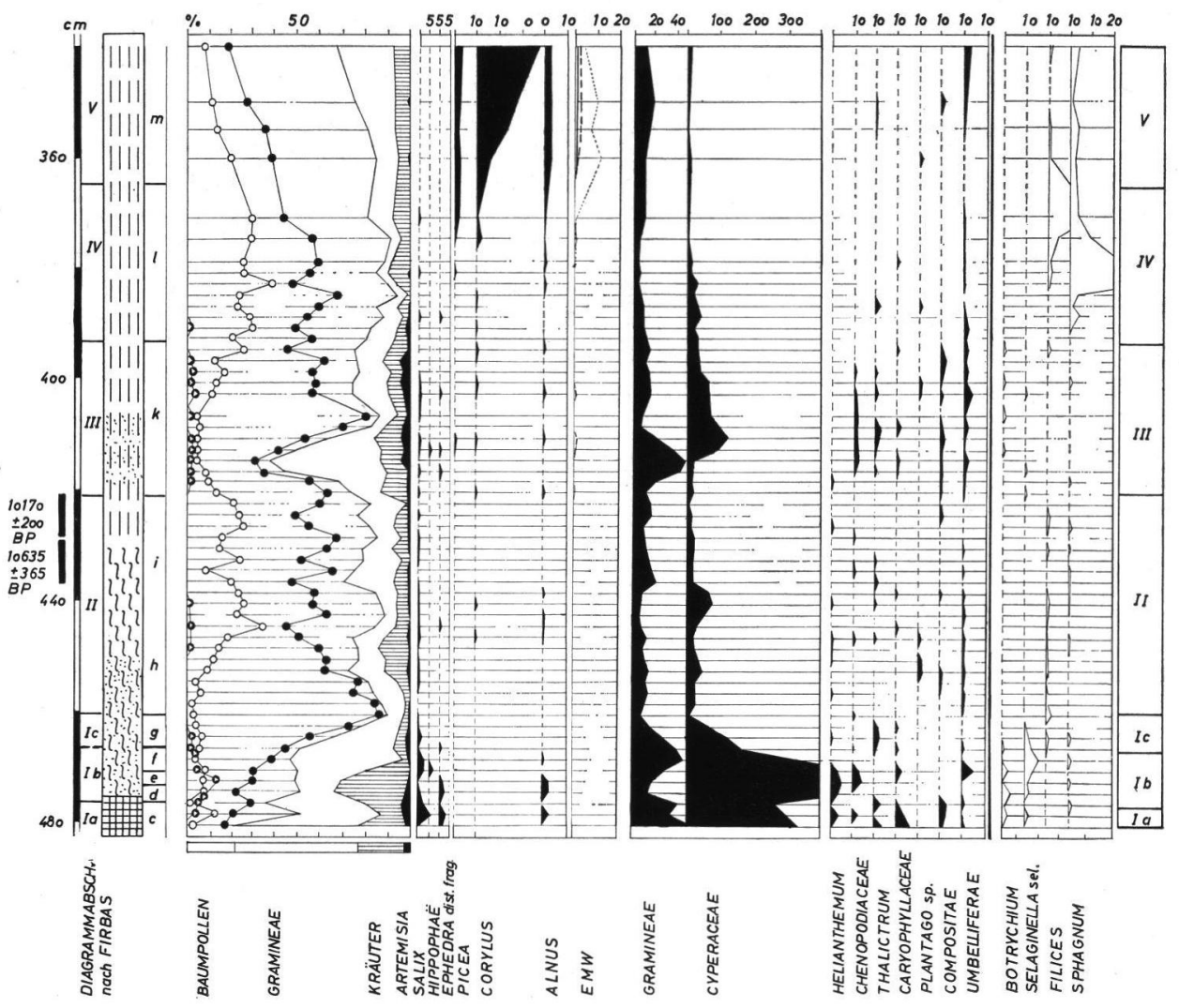

Abb. 4. Pollen- und Sporendiagramm der spätglazialen Moorablagerungen in der Pechschnait bei Traunstein/Obb. Als Gesamtdiagramm berechnet ohne Cyperaceen.

B ewaldungsr ückgang, Abschnitt k: Ein deutlicher Vorstoß der NBP und zwar zunächst der Gramineen und anschließend der Kräuter, von denen besonders Artemisia und die Chenopodiaceen stärker hervortreten, zeigt eine stärkere Lichtung der vorhandenen Wälder an. In den lichtoffenen Beständen breiten sich Juniperus, Hippophaë und Ephedra aus.

$\mathrm{K}$ i e f e r n-Birken z e it mit Spuren wärmeliebender Gehölze.

Abschnitt 1: Die NBP-Vertreter treten zurück, so daß wir wieder eine geschlossene Bewaldung annehmen dürfen. Die Birke hat an der Waldzusammensetzung nun einen stärkeren Anteil (Birkendominanz), aber auch wärmeliebende Arten, wie Corylus, Alnus, Picea und gegen Ende des Abschnittes Vertreter des EMW (besonders die Ulme) erscheinen.

Pinus-Corylus-Zeit, Abschnitt m: Pinus und Betula sind bei starker Ausbreitung der Gräser zurückgedrängt. Gleichzeitig breiten sich die Hasel, aber auch die Ulme, Linde, Eiche und Fichte rasch aus.

\section{Moor bei Moosham}

a) L a ge

Ungefähr $15 \mathrm{~km}$ von Lauter entfernt liegt nördlich des Abtsdorfer Sees bei der Ortschaft Moosham in einer Senke zwischen zwei sich von Südost nach Nordwest erstreckenden Drumlin ein kleines, heute z. T. entwässertes Niedermoor (Abb. 2c). Das Gebiet gehört zu dem sich zwischen Waginger See und Salzach erstreckenden Drumlinfeld (EBERS 1966). Die Senke, in der das Moor eingebettet liegt, ist nach Süden offen. Stratigraphisch 
ist keine Verbindung mit dem Abtsdorfer See zu erkennen. Im ältesten Spätglazial könnte diese Senke, deren Oberfläche heute $440 \mathrm{~m}$ ü. NN liegt, mit dem ehemals größeren Abtsdorfer See in Verbindung gestanden haben. Heute liegt der Wasserspiegel des Sees $12 \mathrm{~m}$ tiefer.

Der Untergrund des Moores besteht aus tonigem Kies, der von blaugrauem Ton, Tongyttja und Kalkgyttjaschichten überlagert wird. Darüber liegt in der Mitte des Moores $130 \mathrm{~cm}$ Niedermoortorf. Am nördlichen Ende des Moores kommt es zu einer lokalen Ausbildung von Lebermudde. Das untersuchte Profil umfaßt nur die Ton- und Kalkgyttja-Schichten. Da wahrscheinlich zwischen der Kalkgyttja und den Niedermoortorfen eine Sedimentlücke besteht, wurden letztere nicht untersucht.2)

b) Stratigraphie

Makrofossilien waren in den Bohrkernen viel weniger enthalten als in Lauter.

bis $130 \mathrm{~cm}$ Niedermoortorf z. T. mit Betula-Holz.

130-195 cm graugelbe Kalkgyttja mit Mollusken. Pinus sp. Holz; Pinus silvestris: Knospenschuppen und Rinde. Betula sp.: Rinde, Reste von Früchtchen; Betula pubescens: bei 175 und $185 \mathrm{~cm}$ je eine Frucht; Betula cf. humilis: bei $177 \mathrm{~cm}$ ein Früchtchen.

195-212 cm grüne, tonige Kalkgyttja. Betula pubescens: 2 Früchtchen. Betula sp.: Teil einer Frucht und einer Fruchtschuppe.

$212-220 \mathrm{~cm}$ graugrüne, kalkhaltige Tongyttja (HF) mit Pyritkügelchen.

$220-230 \mathrm{~cm}$ graugrüne, kalkfreie Tongyttja mit einzelnen Radizellen und unfigurierten $\mathrm{Hu}-$ musteilchen.

c) Vegetationsentwicklung

Die unteren Tongyttja- und Tonschichten wurden nicht untersucht. An Hand des Pollendiagrammes ergibt sich folgendes Bild der Vegetationsentwicklung (Abb. 5):

Waldlose Zeit, Abschnitt c: Die hohen NBP-Werte mit $80 \%$ und die geringe $\mathrm{PD}$, sowie die Sedimente, eine graugrüne, kalkfreie Tongyttja, die vollkommen frei von Großresten ist, weisen auf eine waldlose Zeit hin. Obwohl keine Großreste vorliegen, scheint aber Betula nana in diesem Gebiet stärker verbreitet gewesen zu sein als in Lauter, denn die BetulaWerte liegen viel höher. Betula nana ist übrigens heute noch in dem in der Nähe gelegenen Schönramer Filz heimisch. Pollen von Ephedra, Botrychium und Selaginella konnten für diesen Abschnitt ebenfalls nachgewiesen werden.

Juniperus-Betula-Zeit, Abschnitt d: Ein leichter Rückgang der NBP ist zu verzeichnen. Gleichzeitig erfolgt ein Sedimentationswechsel. Von den Kalkschuttbesiedlern treten besonders Artemisia, Thalictrum und Helianthemum auffällig zurück. Juniperus und Betula cf. nana breiten sich aus.

Abschnitt e: Gramineen, Cyperaceen und Kräuter treten in diesem Abschnitt merklich zurück. Dafür breitet sich Juniperus aus (Maximum 19\%). Wie in der Pechschnait geht der Juniperus-Dominanz eine solche der Weide voran. Hippophaë ist bis $2 \%$ vertreten. Im Gegensatz zu Lauter breitet sich die Birke schon vor dem Juniperus-Maximum aus. Mit dieser Strauchphase setzt die Wiederbewaldung ein.

Betula-Zeit, Abschnitt f: Juniperus wird durch eine kräftige Ausbreitung der Birke zurückgedrängt. Im weiteren Verlauf dringt die Kiefer vor, wobei die NBP-Werte nur langsam zurückgehen.

Abschnitt g: Der im vorangehenden Abschnitt erkenntliche Vorstoß der Kiefer wird plötzlich gestoppt. Kiefern- und Birkenkurve bleiben während des gesamten Abschnittes fast unverändert. Ein leichter Anstieg von Artemisia ist erkennbar, auch ein Rückgang der PD zu verzeichnen. Mit Beginn dieses Abschnittes erfolgt auch die Bildung tonfreier Kalkgyttja.

$P$ in us-Betula-Zeit, Abschnitt h: Zunächst dominiert noch die Birke; im weiteren Verlauf tritt aber die Kiefer stärker hervor. Es ist die Zeit der geschlossenen Bewaldung, denn die NBP-Werte sind auf $10 \%$ abgesunken. Von den Sträuchern ist zwar Juniperus noch in einzelnen Spektren vorhanden, aber immer nur mit sehr geringen Werten.

2) Von der Abteilung Moorkunde und Torfwirtschaft der Bayer. Landesanstalt für Bodenkultur, Pflanzenbau und Pflanzenschutz wurde ein Längs- und Querprofil durch das Moor gelegt; für die Überlassung der Unterlagen wird gedankt. 
$P$ inus-Zeit, A bschnitt i: Bei weiterer Zurückdrängung der Birke dor Miert jetzt die Kiefer. Die Juniperus-Werte sind höher als früher. Vereinzelte Pollen von Corylus und Ephedra sind vorhanden. Da auch ein leichter Anstieg der NBP-Werte zu verzeictenen ist, kam es wahrscheinlich zu einer Auflockerung der geschlossenen Bewaldung.

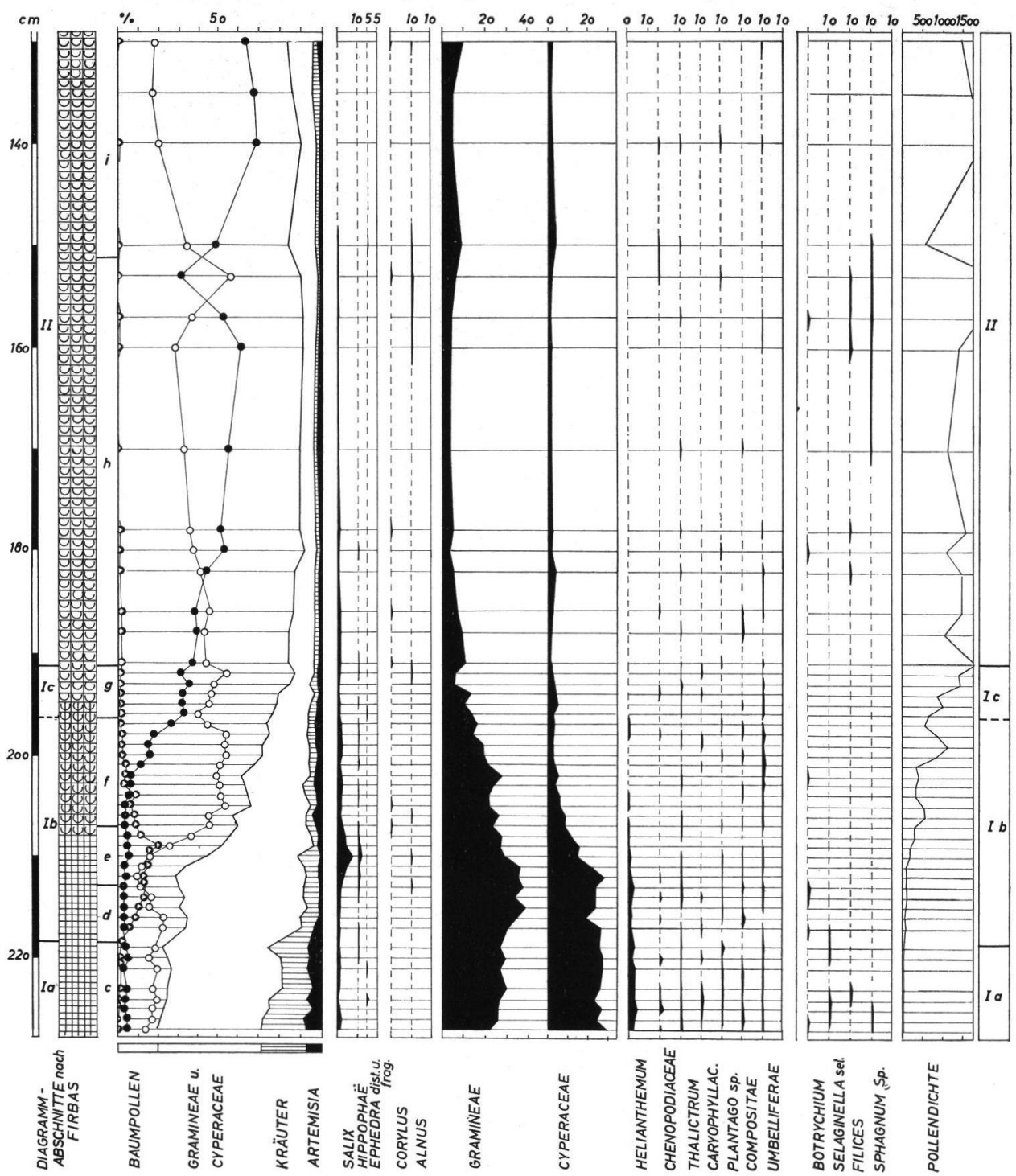

Abb. 5. Pollen- und Sporendiagramm des Spätglazials von Moosham am Abtsdorfer See. Als Gesamtdiagramm berechnet.

\section{Diskussion und Datierung}

Das Profil von tuter reicht von allen drei untersuchten Profilen am weitesten zurück, denn in der T :umlinlandschaft wurden die ältesten Ablagerungen nicht untersucht, und in der Pechsc ${ }^{3}$, ait setzte die Moorbildung im untersuchten Profil erst später ein. Die Boden- und Kli: sverhältnisse der drei Gebiete sind zwar verschieden, aber die Vege- 
tationsentwicklung weist viele gemeinsame Züge auf. Kurz zusammengefaßt können wir folgende Zeitabschnitte unterscheiden:

1. Die waldlose Zeit, die die Diagrammabschnitte $a, b$ und c umfaßt. Die ersten beiden Abschnitte sind nur aus dem Randmoränengebiet bei Lauter bekannt, während der Abschnitt $\mathrm{c}$ auch schon in den beiden anderen Profilen enthalten ist. Der NBPAnteil ist sehr hoch, die Pollendichte (PD) gering. Vorherrschend sind an Gramineen, Cyperaceen und Artemisia reiche Pioniergesellschaften, welche die Rohschuttböden besiedelten. In den ältesten Ablagerungen sind die Pinuspollen wahrscheinlich auf Fernflug zurückzuführen. Kriechweiden, Betula nana und Ephedra-Bestände waren schon vorhanden.

Im Abschnitt c kommt es zu einem Rückgang von Artemisia und anderen Pionierpflanzen. In der Drumlinlandschaft sind die Birkenwerte hoch, und in Lauter sprechen Rindenreste und Holzelemente der Birke für größere Betula nana Bestände in der Nähe des Endmoränen Sees. Juniperus ist in diesen Abschnitten auch schon stärker vertreten. In der Pechschnait erreicht die Kiefer im Abschnitt c Pollenwerte, die auf Vorkommen derselben, wahrscheinlich als Pinus montana, in der Nähe des Moores schließen lassen.

Für die waldlose Zeit liegen also im untersuchten Gebiet die gleichen Vegetationsverhältnisse vor, wie sie schon von Bertsch (1961), Beug, Burger (1964), LANG (1952), LÜRZER (1954) und WELTEN (1952) für das übrige Alpenvorland gefunden wurden. Diese waldlose Zeit können wir der ältesten Tundrenzeit (Ia nach FIRBAs) gleichsetzen.

2. Zeit der Wiederbewaldung. Sie umfaßt die Abschnitte d-g. Der Diagrammabschnitt $\mathrm{d}$ ist in allen drei Profilen gekennzeichnet durch den Ausklang der Pionierpflanzen (Artemisia, Helianthemum, Thalictrum u. a.), die durch eine Strauchphase abgelöst werden. Neben Weiden breitet sich besonders Juniperus aus. In der Drumilin- und Randmoränenlandschaft kommt aber auch noch Hippophä dazu. Auf Grund der Pollenwerte haben aber die Hippophä̈-Bestände nie die Ausdehnung erreicht wie in anderen Gebieten (siehe Zusammenstellung bei Firbas 1949, BERTSCH 1961 (Pfarrmoos und Buchensee), Paul und Lutz 1939).

In vielen Profilen des SW-deutschen Alpenvorlandes (BERTSCH 1961, LANG 1952) kommt es zu Beginn des Juniperus-Anstieges zu einer Pinus-Depression, die auch in den beiden Profilen von Lauter und vom Abtsdorfer See in Erscheinung tritt. Dagegen ist in der Pechschnait ein Anstieg der Pinus-Werte schon im Abschnitt d zu verzeichnen, dessen Ursache aber nicht genügend geklärt werden konnte. Zu einem Rückgang der Birkenwerte und einem darauf folgenden Anstieg kommt es in Lauter und in der Pechschnait zur Zeit des Juniperus-Gipfels. In der Drumlinlandschaft breitet sich die Birke schon vor dem Juniperus-Maximum aus. Der Birkenvorstoß im Anschluß an die Juniperus-Hippophä̈-Strauchphase erfolgt durch Baumbirken. In der Pechschnait ist derselbe nur angedeutet, dafür breitet sich aber die Kiefer kräftig aus. Während es in der Randmoränenlandschaft von Lauter und in der Pechschnait zu einer weiteren Ausbreitung der Kiefer kommt, bei der die Birke besonders stark zurückgedrängt wird, ist in der Drumlinlandschaft eine deutliche Stagnation der Kiefer erkennbar (Abschnitt g). In allen drei Profilen treten zu dieser Zeit erneut Ephedra und Hippophaë auf. In der Pechschnait ist sogar ein leichter Anstieg der ArtemisiaWerte erkennbar. Die einzelnen Phasen der Wiederbewaldung stimmen mit denen aus dem südwestdeutschen Alpenvorland (Bertsch u. LANG) und aus dem Gebiet des ehemaligen Rosenheimer Sees gut überein (BEUg mündlich), dagegen nicht ganz mit denen aus dem östlich der Salzach liegenden Gebieten. Hier tritt nämlich Juniperus 
zur Zeit der Wiederbewaldung fast vollständig zurück, wie aus den Profilen von Lürzer aus dem Egelseegebiet und aus den Lunzer Seen von Burger hervorgeht. Die Strauchphase vor dem Baumbirkengipfel ist aber dafür im Egelseegebiet und im Ibmer Moos (Pammer-Gräflinger in Gams 1947) durch hohe Hippophä̈-Werte gekennzeichnet.

3. Zeit der geschlossenen Bewaldung. Eine geschlossene Bewaldung darf in Abschnitt h, in dem in allen drei Profilen die NBP-Werte unter $15 \%$ abgesunken und die Vertreter lichtoffener Gesellschaften weitgehend verschwunden sind, angenommen werden. In der Pechschnait und in Lauter sind es Kiefernwälder, denn Großreste aus dieser Zeit von Pinus silvestris sind reichlich vorhanden; in der Drumlinlandschaft dagegen überwiegt die Birke. Das Verhältnis Betula-Pinus scheint auch in unserem Gebiet denselben Schwankungen unterworfen geweren zu sein, wie in Südwestdeutschland.

Gegen Ende des Abschnittes h kommt es in der Pechschnait zu einer deutlich hervortretenden Birkendominanz, die mit einer Reiserschicht von Baumbirken zusammenfällt. Es muß zu einem Stillstand des Moorwachstums gekommen se:n, die eine Birkenausbreitung auf dem Moor ermöglichte, deren Ursache nicht zu erkennen ist. Da stärkere Birkenstämme fehlen, kann die Zeit nur kurz gewesen se:n. Im nahe gelegenen Lauter und am Abtsdorfer See ist diese Birkenausbreitung nur angedeutet. Da in allen drei Profilen in den Spektren dieses Abschnittes auch Pollen wärmeliebender Sträucher und Bäume auftreten (Corylus und Alnus), wenn auch nur in Pollenwerten, die höchstens auf einen Weitflug schließen lassen, so könnte an eine Erwärmung gedacht werden. Durch dieselbe könnten die Einwanderungsgeb:ete von Corylus und Alnus näher an das Untersuchungsgebiet herangerückt sein. Auch BEUG fand im Gebiet des ehemaligen Rosenheimer Sees in entsprechenden Diagrammabschnitten wärmeliebende Gehölzpollen. Andererse:ts ist aber auch der Gedanke, daß Pollen aus dem Gebiet südlich des Alpenhauptkammes stammen und durch Föhn in das Untersuchungsgebiet verfrachtet wurden, nicht ganz auszuschließen.

4. Lichtung der Wälder. Mit einem Zurücktreten der Birken und einer erneuten Zunahme der Gräser enden die Profile von Lauter und vom Abtsdorfer See (Abschnitt i). In der Pechschnait ist dieser Abschnitt durch ein vermehrtes Auftreten der NBP gekennzeichnet.

Der starke Anstieg bis zu 60\% im Diagrammabschnitt $\mathrm{k}$ in der Pechschnait läßt auf eine starke Lichtung der Wälder in den Höhenlagen um $700 \mathrm{~m}$ schließen. An Artemisia reiche Gramineen-Bestände spielen wieder eine Rolle, Strauchgesellschaften mit Salix, Hippophä̈, Juniperus und Ephedra breiten sich aus, Botrychium und Selaginella und andere Vertreter lichtoffener Standort sind wieder vertreten. In den Torfen dieses Abschnittes konnte, wenn auch nur mikroskopisch, eine Anreicherung von Quarzsand nachgewiesen werden. Daraus kann auf eine erneute Klimadepression geschlossen werden. Wenn gleichzeitig der Pollen von Corylus, Alnus, Picea und Tilia in einzelnen Spektren erscheint, so kann dies nur durch Fern- oder Weitflug bedingt sein, deren Niederschlag jetzt in den waldfreien Gesellschaften natürlich stärker hervortritt.

5. W i e de rbew a ldung. Aus dem sich nun anschließenden Rückgang der NBP und des Wacholders ist eine Zunahme der Bewaldungsdichte im Abschnitt 1 auch in den Höhenlagen um $700 \mathrm{~m}$ zu ersehen. Neben Picea, Corylus und Alnus erscheinen nun die Vertreter des EMW. Die Birke, die zur Zeit der Lichtung der Wälder fast ganz zurückgedrängt wurde, breitet sich nun wieder aus. Durch einen raschen Vorstoß der Hasel und der EMW-Komponenten, besonders der Ulme, ist der folgende Abschnitt gekennzeichnet. 
Die ersten Diagrammabschnitte $(\mathrm{a}-\mathrm{c})$ wurden schon der ältesten Tundrenzeit, Ia nach Firbas (1949) zugeordnet. Es soll nun versucht werden, die anderen lokalen Abschnitte in die Spätglazialchronologie einzuordnen.

Der Nachweis des für das Alleröd wichtigen Leithorizontes, der Laacher Tuffschicht, war nicht gegeben. Es war daher nur eine Datierung durch Fernkonnektierung mit einwandfrei datierten Diagrammen aus dem Südwestdeutschen Alpenvorland möglich. Nach FAEGRI (1939/40) muß als wichtigster Leithorizont des Spätglazials die Klimadepression der jüngeren Dryaszeit (III nach Firbas) angesehen werden, deren Nachweis für Südwest-Deutschland (Bertsch, A., 1961, LANG, 1952), die Vogesen (Firbas u. Mitarbeiter, 1948), den Schwarzwald (LANG, 1954) und das alpine Vereisungsgebiet (Welten, 1952, ZAGWJIN, 1952), sowie für das östl. Salzachgletschergebiet (LüRZER, 1954) gesichert ist. Neben dem Rückgang der Bewaldung kann als weiteres Kriterium für die Zuordnung zur jüngeren Dryaszeit auf Grund der Untersuchungen von A. BERTsch die erneute Ausbreitung von Juniperus herangezogen werden. Ein Sedimentationswechsel kennzeichnet außerdem in vielen Fällen den Übergang vom Alleröd zur jüngeren Dryaszeit, bzw. von dieser zum Präboreal.

Da nun in der Pechschnait der Rückgang der Bewaldung, der auf eine Klimaverschlechterung schließen läßt, vor der Ausbreitung wärmeliebender Arten gut erkennbar ist und auch andere Befunde (erneute Ausbreitung von Juniperus und Sed:mentationswechsel in der Stratigraphie dieses Abschnittes) dafür sprechen, kann der Abschnitt k unseres Diagrammes eindeutig der jüngeren Dryaszeit (III nach FIRBAs) zugeordnet werden.

$\mathrm{Ob}$ der in Lauter am Ende des lokalen Abschnittes i angedeutete NBP-Vorstoß, verbunden mit einem Sedimentationswechsel, dem Bewaldungsrückschlag in der Pechschnait, also dem Beginn der jüngeren Dryaszeit entspricht, möge entgegen früheren Feststellungen (Schmeide, 1970) einstweilen dah:ngestellt bleiben. Wir wissen zwar durch die Untersuchungen aus Südwest-Deutschland und der Schweiz, daß in tieferen Lagen die Lichtung der Wälder nicht so einschneidend sich abzeichnete wie in Höhen über $700 \mathrm{~m}$. Firbas (1934) konnte in Kolbermoor - die neuen Untersuchungen von Beug bestätigen dies keinen ausgeprägten Klimarückschlag feststellen. $\mathrm{Ob}$ in Lauter und am Abtsdorfer See die Klimadepression der jüngeren Dryaszeit eine Lichtung der Wälder bedingte, könnten erst weitere Profile aus diesen Gebieten klären. Im östlichen Salzachgletschergebiet, im Gebiet der Egelseen $(600 \mathrm{~m})$, zeichnet sich jedenfalls die jüngere Dryaszeit mit einer Lichtung des Waldbestandes sehr deutlich ab (Lürzer, 1954). Hier fallen ein starker NBPAnstieg und ein scharfer Sedimentat:onswechsel mit einem Birkenvorstoß zusammen, während im Gebiet der Pechschnait $(700 \mathrm{~m})$ sich in dieser Zeit eine ausgesprochene BetulaDepression abzeichnet.

Die vor der 2. Dryaszeit liegenden Abschnitte h und i, die am Abtsdorfer See und in Lauter bei niedrigen NBP-Werten und hoher Pollendichte auf geschlossene Wälder schließen lassen, in denen in Lauter die Kiefern vorherrschen, im Drumlingebiet die Birken einen größeren Anteil haben, müssen demnach dem Alleröd (II) entsprechen. In der Pechschnait gehören diese beiden lokalen Abschnitte ebenfalls dieser Zeit an. Hier scheint nur die Bewaldung nicht so geschlossen gewesen zu sein wie in den tieferen Lagen. Stratigraphisch ist die Allerödzeit in der Randmoränen- und Drumlinlandschaft durch Bildung einer tonfreien Kalkgyttja gekennzeichnet, während in der Pechichnait hauptsächlich Braunmoostorfe gebildet wurden.

Zuzuordnen bleiben nur noch die zwischen der waldlosen Zeit (Ia) und dem Alleröd liegenden Abschnitte d bis g. Mit dem Nachweis von Juniperus in den dänischen Spätglazialprofilen durch Iversen (1934 und 1946) und im Alpenvcrland durclı A. BertSCH (1961), haben wir klarere Vorstellungen über die erste Phase d :r Wiederbewaldung gewonnen. Die Hippophä̈-Werte sind, wie z. B. in unseren Diag anmen, ofl: sehr niedrig 
und der Nachweis einer Strauchgesellschaft vor dem Auftreten von Baumbirken- und Kiefernbeständen war dadurch oft problematisch. Die Juniperus-Kurve dagegen ermöglicht eine genaue Grenzziehung und eine Zuordnung einzelner Vegetationsabschnitte. Der Juniperus-Anstieg fällt in unseren Diagrammen mit dem starken Rückgang der NBPWerte, besonders mit dem der Gramineen und der Helianthemum und Thalictrum reichen Gesellschaften zusammen. Zur Zeit des Wacholdergipfels deuten höhere Hippophaë-Werte auf eine stärkere Ausbreitung des Sanddorns. Dem Juniperus-Maximum eilt in allen drei Profilen ein Weidengipfel ein wenig voraus. Die ausgedehnten Salix-, Juniperus- und Hippophä̈-Bestände werden anschließend durch sich rasch ausbreitende Baumbirken- und Kiefernbestände verdrängt. Diese Vegetationsabschnitte stehen ganz in Übereinstimmung mit den am Bodensee und in Oberschwaben gefundenen Ergebnissen und wir dürfen diese Abschnitte $(\mathrm{d}-\mathrm{g})$ dem Bereich I bc (nach FIRBAs) zuordnen. In der Pechschnait liegt zwar der Kieferanstieg noch vor dem Juniperus-Maximum, das wir aber dem in Lauter gleichsetzen können. Ob hier in den ehemals eisfreien Gebieten schon vor der Ausbreitung von Juniperus Pinus mugo in lockeren Beständen vorhanden war, kann nur vermutet werden.

Wie in den meisten Diagrammen vom Bodensee (LANG, 1952), BERTSCH, 1961), in Oberschwaben (GötTlich, 1960), dem Schwarzwald (LANG, 1954) und der Schweiz (Welten, 1952) ist auch in den vorliegenden Profilen keine klare Gliederung in eine progressive Phase der Böllingzeit (Ib) und eine regressive der älteren Tundrenzeit (Ic) vor Beginn der Allerödzeit möglich. Auch die neuen Untersuchungen von BEUG aus den Kolbermoor und vom Simssee (Gebiet des ehemaligen Rosenheimer Sees) lassen keinen einwandfreien Klimarückschlag in diesen Abschnitten erkennen. In dem Diagramm von Lürzer aus dem Egelseegebiet könnte die stärkere Ausbreitung der Gramineen in dem als I c bezeichneten Abschnitt auf eine Klimaverschlechterung zurückzuführen sein. BEUG fand zwar auch im Kolbermoor nach dem Juniperus-Gipfel einen deutlichen Gramineenvorstoß, der vielleicht mit einer Klimadepression zusammenhängen könnte. In dem nicht weit entfernten Simssee dagegen ist derselbe nur ganz schwach angedeutet. In unseren Diagrammen von Lauter und vom Abstdorfer-See ist im Abschnitt $f$, zur Zeit der Birkendominanz, ein leichter Anstieg der Artemisia- und Kräuterpollenwerte zu verzeichnen. Damit könnte eine regressive Vegetationsentwicklung angedeutet sein. Nach LANG (1952) dürfte die alpine Waldgrenze gegen Ende der Böllingzeit nahe bei $700-800 \mathrm{~m}$ gelegen haben. Der Klimarückschlag müßte sich also im Diagramm der Pechschnait klar abzeichnen. Es kommt hier zwar nach dem Juniperus-Gipfel zur erneuten Ausbreitung von Weiden und grasreicheren Gesellschaften, die auf einen Klimarückschlag schließen lassen, ein einwandfreier Beweis ist aber mit dem vorhandenen Profil nicht zu erbringen. Untersuchungen in kleineren Abständen und Profile aus höheren Lagen würden vielleicht einen Nachweis von I c ermöglichen.

Neben der Fernkonnektierung der Pollendiagramme kann auch noch die Radiokarbon-

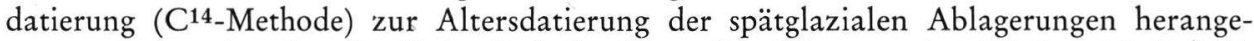
zogen werden. Aus dem Profil der Pechschnait wurden daher aus dem lokalen Abschnitt i, der noch dem Alleröd zugerechnet wird, 2 Proben vom Niedersächsischen Amt für Bodenforschung in Hannover auf ihr C14-Alter hin bestimmt. Das Ergebnis lautet: die unmittelbar unter dem NBP-Vorstoß abgelagerten Seggentorfe $(420-430 \mathrm{~cm})$ ergaben ein Alter von (Hv 1247) $10170 \pm 200$ Jahren BP. Für die darunterliegenden $10 \mathrm{~cm}$ Braunmoostorfe $(430-440 \mathrm{~cm})$ wurde ein Alter von $(\mathrm{Hv} 1248) 10635 \pm 365$ Jahren BP ermittelt (freundliche briefl. Mitteilung von Herrn Dr. GeY).

Für die Pollenzone II (Alleröd) wird von Gross (1954) auf Grund zahlreicher C14_ Datierungen der Zeitraum von 11900-10750 Jahren BP angegeben. Für die jüngere Dryaszeit (III) gibt er den Zeitraum von 10750-9950 Jahren BP an. Zwischen dem 
Ergebnis der C14-Datierung und dem der Vegetationsentwicklung besteht damit keine Übereinstimmung. Die jüngere Probe (Hv 1247) ist nämlich vegetationsgeschichtlich älter als die II. Tundrenzeit (10750-9950 Jahre BP). Auf Grund der C14-Datierung in Hannover ist sie aber um einige Jahrhunderte jünger. Erst bei einer Wahrscheinlichkeit von $99 \%$ würde die untere Grenze der älteren Tundrenzeit gerade noch erreicht werden. Die Gründe für diese Differenz sind uns nicht bekannt. Es besteht nur die Möglichkeit, daß durch die Einsickerung von Wasser in Spalten jüngere Humusstoffe in die Allerödschichten gelangten und damit das wahrscheinliche $\mathrm{C}^{14}$-Alter dieser Schichten verfälscht wurde.

\section{Zum Auftreten wärmeliebender Pollen}

In allen drei Profilen konnten, beginnend schon mit den ältesten Ablagerungen, immer wieder Pollen wärmeliebender Bäume und Sträucher, besonders Corylus, Alnus und Picea nachgewiesen werden. Es handelt sich hier zweifellos um Fern- oder, in den jüngeren Schichten, um Weitflugpollen (FIrbas 1949). Lürzer, deren Profil keine $40 \mathrm{~km}$ von Lauter entfernt ist, hat vor dem Hippophä̈-Gipfel, der mit unserer Juniperus-Hippophaë-Phase in I bc identisch ist, zwei kleine Fichtengipfel von 2,5\% gefunden. Für diesen Zeitraum war kein Nachweis von Picea in unseren Spektren möglich; dafür erscheinen aber Picea-Pollen sowohl in Lauter als auch am Abtsdorfer See in unserem lokalen Abschnitt c, der noch der waldlosen Zeit angehört. Daß die Fichte schon vor dem Alleröd von Osten gegen Westen vorrückte, beweisen die Pollenfunde vom Lunzersee vom Gams (1927) und Burger (1964) im Alleröd bzw. in Ibc. Das vereinzelte Vorkommen von Pollen wärmeliebender Pflanzen in verschiedenen Diagrammabschnitten vor dem Präboreal erlaubt aber nicht die Annahme, daß im Voralpen- oder alpinen Raum wärmeliebende Arten schon vor dieser Zeit Fuß gefaßt hätten.

\section{Das Vorkommen von Ephedra}

Das von Mayer $(1964,1965$, 1966) für Bayern erstmals nachgewiesene Vorkommen von Ephedra und zwar vom distachya- und fragilis-Typ konnte auch in zahlreichen Spektren der untersuchten Profile bestätigt werden. Der Erhaltungszustand der Pollen war durchwegs gut. Pollenkörner von Ephedra wurden in den Spektren Ia, Ibc und III allgemein gefunden. In der Pechschnait wurde der Pollen von Ephedra distachya in II und an der Wende von III/IV nachgewiesen. Der Pollen vom fragilis-Typ trat hauptsächlich in Ia und Ibc auf. Ephedra war sowohl auf den lichtoffenen Standorten des Spätglazials des alpinen Gebietes (ZAGwJIN 1952, Welten 1957, Burger 1964) als auch im gesamten Alpenvorland (Lang 1952, Bertsch, A. 1961, Göttlich 1955, 1960, Gronbach 1960) und im periglazialen Gebiet, weit außerhalb der alpinen Vereisung in der Oberpfalz (Schmeide 1969) weit verbreitet.

\section{Zur Molluskenfauna des spätglazialen Sees bei Lauter}

In der Kalkgyttja des spätglazialen Randsees von Lauter waren Mollusken ziemlich häufig vertreten. Eine Bestimmung derselben ergab, daß es sich um Arten handelt, die im Schlamm stehender oder schwach bewegter Gewässer leben. Über die Wassertemperatur oder gar über das Klima geben sie leider keine Auskunft, da es sich bei den bestimmten Arten um euryöke Würmüberdauerer handelt, die im Würmperiglazial in Mitteleuropa lebten. ${ }^{3}$ ) Die Bestimmung ergab, daß es sich nur um 2 Schneckenarten handelte und zwar um Valvata piscuides OF Müller und Valvata pulchella StUder. Außerdem waren noch Schalen von Pisidium sp. vorhanden.

Erst nach Drucklegung war mir die Arbeit von KLAus, W.: Pollenanalytische Untersuchungen zur Vegetationsgeschichte Salzburgs: Das Torfmoor am Walser Berg, Verh. d. Geol. Bundesanstalt, H. 1/2, 200-212, 1967 zugänglich, deren Ergebnisse eine gute Übereinstimmung mit den vorliegenden Untersuchungen zeigen.

\section{Literaturverzeichnis}

Beug, H.-J.: Leitfaden der Pollenbestimmung. - Fischer Verlag, Stuttgart 1961.

- : Die spätglaziale und frühpostglaziale Vegetationsgeschichte im Gebiet des ehemaligen Rosenheimer Sees (Oberbayern). - Unveröffentlicht.

3) Herrn Dr. Ant, von der Bundesanstalt für Naturschutz und Landschaftspflege, Bad Godesberg, möchte ich hiermit für die Bestimmung der Mollusken nochmals meinen herzlichsten Dank aussprechen. 
BęrTsCH, A.: Untersuchungen zur spätglazialen Vegetationsgeschichte Südwestdeutschlands. Flora, 151, 243-280, Jena 1961.

Burger, D.: Results of a pollenanalytic investigation in the Untersee near Lunz in Austria. Geologie en Mijnbouw, 43, 94-102, 1964.

Ebers, E., Weinberger, L., Del Negro, W.: Der pleistozäne Salzachvorlandgletscher. - Veröff. d. Ges. f. Bayer. Landeskunde, H. 19-22, München 1966.

Erdtman, G., Berglund and Praglowski, J.: An introduction to a scandinavian flora. - Bd., I. u. II., Almquist \& Wiksell, Stockholm 1961.

Erdtman, G.: An introduction to pollenanalysis. - Waltham, Mass. USA 1954.

Faegri, K. u. Iversen, J.: Textbook of pollen analysis. - Blackwell, Oxford 1964.

Firbas, F.: Pollenanalytische Untersuchungen einiger Moore der Ostalpen. - Lotos, 71, 187242, Prag 1923.

- : Die Vegetationsentwicklung des mitteleuropäischen Spätglazials. - Bibl. Botanica, 112, $1-68,1935$.

- : Über das Verhalten von Artemisia in einigen Pollendiagrammen. - Biol. Zbl., 67, 17-28, 1949.

— : Waldgeschichte Mitteleuropas. - Bd., I u. II, Fischer, Jena, 1949/52.

- : Die Synchronisierung der mitteleuropäischen Pollendiagramme. - Danm. Geolog. Unders. II, Roekke, 80, 12-21, 1954.

Gams, H.: Die Geschichte der Lunzer Seen, Moore und Wälder. - Intern. Revue d. ges. Hydrobiol. u. Hydrogeographie, 18, 305-387, 1927.

- : Das Ibmer Moos. - Jb. d. O. Ơ. Musealver., 92, 1-48, Linz 1947.

GöтtцICH, Kh.: Ein Pollendiagramm ungestörter späteiszeitlicher Verlandungsschichten im Federseebecken. - Beitr. z. naturkdl. Forsch. in SW-Deutschland, 14, 88-92, 1955.

- : Beiträge zur Entwicklungsgeschichte der Moore in Oberschwaben. - Jh. Vaterl. Naturkd. Württemberg, 115, 93-174, Stuttgart 1960.

Gronbach, G.: Pollenanalytische Untersuchungen zur Geschichte des Federsees und zur vorgeschichtlichen Besiedlung des Federseerieds. - In: Der „Federsee“, Herausgeber W. Zimmermann, 316-355, Tübingen 1960.

Gross, H.: Das Alleröd-Interstadial als Leithorizont der letzten Vereisung in Europa und Amerika. - Eiszeitalter u. Gegenwart, 4/5, 189-209, O'hringen 1954.

- : Die bisherigen Ergebnisse von C14-Messungen und paläolithische Untersuchungen für dic Gliederung und Chronologie des Jungpleistozäns in Mitteleuropa und den Nachbargebieten. - Eiszeitalter u. Gegenwart, 9, 155-187, Ohringen 1958.

Krisar, R.: Das Filzmoos bei Tarsdorf in Oberösterreich. - Phyton, 9, 217-251, Horn, Austria 1961.

LANG, G.: Zur spätglazialen Vegetations- und Florengeschichte Südwestdeutschlands. - Flora, 139, 243-294, Jena 1952.

- : Chronologische Probleme der späteiszeitlichen Vegetationsentwicklung in SW-Deutschland und im franz. Zentralmassiv. - Pollen et Spores, v, 130-142, Paris 1963.

LürZER, E.: Das Spätglazial im Egelseegebiet (Salzach Vorland-Gletscher). - Zeitschr. f. Gletscherkde. u. Glazialgeologie, III, 83-90, Innsbruck 1954.

Mayer, H.: Praeboreale Vorkommen von Ephedra (Meerträubl) auf dem Steinernen Meer (Berchtesgadner Kalkalpen). - Naturw., 51, 343/344, 1964.

- : Tannenreiche Wälder am Nordabfall der mittl. Ostalpen. Vegetationsgefälle in montanen Waldgesellschaften von den Chiemgauer und Kitzbühler Alpen zu den nördl. Hohen Tauern, - Zillerntaler Alpen, München 1963.

- : Zur Waldgeschichte des Steinernen Meeres. - Jb. Ver. z. Schutze d. Alpenpfl. u. Tiere, 30, 100-120, München 1965.

- : Waldgeschichte des Berchtesgadner Landes. - Forstw. Forsch., Beih. z. Forstw. Cb., 22, 1966.

Paul, H. u. Ruoff, S.: Pollenstatistische und stratigraphische Mooruntersuchungen im südl. Bayern. I. Teil: Moore im außeralpinen Gebiet der diluvialen Salzach-, Chiemsee- und Inngletscher. - Ber. Bayer. Botan. Ges., 29, 1-84, München 1927.

Rabien, I.: Zur Bestimmung fossiler Knospenschuppen. - Paläontol. Zeitschr., 27, 57-66, 1953.

SchmeIdL, H.: Zur Altersdatierung der Mettenhamer Filze. - Erläuter. z. Geolog. Karte von Bayern, Bl. Nr. 8240, Marquartstein, 170-174, München, 1967.

- : Beitrag zur spätglazialen Vegetations- und postglazialen Waldentwicklung im südl. Oberpfälzer Wald. - Erl. zur Bodenkarte von Bayern, Blatt Nr. 6640, Neunburg vorm Wald, 103-113, München 1969.

- : Die spätglaziale Vegetationsentwicklung im westl. Salzachgletschergebiet. - Mitt. d. Ostalp. Din. Pflanzensoziol. Arbeitsgem., 10/2, 70-72, Wien 1970.

Troels-Smith, J.: Pollenanalytische Untersuchungen zu einigen schweizerischen Pfahlbauproblemen. - In: Das Pfahlbauproblem, 11-58, Birkhäuser Verl. Basel 1955. 
Vidal, H. u. Hohenstatter, E.: Die Moore des Kartenblattes Oberteisendorf. - Unveröffentlicht.

Welten, M.: Pollenanalytische, stratigraphische und geochronologische Untersuchungen am Faulenseemoos bei Spiez. - Veröff. Geobotan. Inst. Rübel, 21, 1-201, Zürich 1944.

— : Uber die spät- und postglaziale Vegetationsgeschichte des Simmentales. - Veröffentl. d. Geobotan. Inst. Rübel, 26, 1-133, Zürich, 1952.

- : Uber das postglaziale und spätglaziale Vorkommen von Ephedra am nordwestl. Alpenrand. - Ber. d. Schw. Botan. Ges., 67, 33-54, 1957.

ZAGwJIN, W.: Pollenanalytische Untersuchungen einer spätglazialen Seeablagerung aus Tirol. Geol. en Mijnbouw, serie 7, 14, 235-239, 1952.

Manuskr. eingeg. 9. 2. 1971.

Anschrift des Verf.: Dr. Hans Schmeidl, D 8214 Bernau/Chiemsee, Moorforschungsstelle der Bayer. Landesanstalt für Bodenkultur, Pflanzenbau und Pflanzenschutz. 